\title{
Erratum: Towards the understanding of the origin of charge-current-induced spin voltage signals in the topological insulator $\mathrm{Bi}_{2} \mathrm{Se}_{3}$ [Phys. Rev. B 92, 201102(R) (2015)]
}

\author{
E. K. de Vries, A. M. Kamerbeek, N. Koirala, M. Brahlek, M. Salehi, S. Oh, B. J. van Wees, and T. Banerjee
} (Received 3 April 2016; published 27 April 2016)

DOI: 10.1103/PhysRevB.93.159907

We have found an error in our publication regarding the direction of the arrow that represents the direction of the surface state spin polarization $\sigma$. According to the convention as used in our paper, the direction of $\sigma$ in Figs. 2(c), 5(c), and S1(c) should be flipped to the $+y$ direction (i.e., those should be opposite to that of Figs. 2(b), 5(b), and S1(b)) as the current direction is inverted. This follows from the spin-momentum locking relation that holds for the surface states in our topological insulator $\mathrm{Bi}_{2} \mathrm{Se}_{3}$.

This change does not affect any conclusions made in our paper.

We thank Dr. Connie Li of Naval Research Laboratory, USA, for pointing this out. 